Biochemistry. 2007 May 15; 46(19): 5732-5740. doi:10.1021/bi700300u.

\title{
Crystal structure of KPC-2:
}

\section{insights into carbapenemase activity in Class $A$-lactamases}

\author{
Wei Ke ${ }^{1}$, Christopher R. Bethel ${ }^{2}$, Jodi M. Thomson ${ }^{3}$, Robert A. Bonomo ${ }^{2,3}$, and Focco van \\ den Akker ${ }^{1}$ \\ 1Department of Biochemistry, Case Western Reserve University, Cleveland, Ohio 44106
}

2Research Service, Louis Stokes Cleveland Veterans Affairs Medical Center, Cleveland, Ohio 44106

3Department of Pharmacology, Case Western Reserve University, Cleveland, Ohio 44106

\begin{abstract}
$\beta$-lactamases inactivate $\beta$-lactam antibiotics and are a major cause of antibiotic resistance. The recent outbreaks of $\underline{\text { Klebsiella }}$ pneumoniae carbapenem-resistant (KPC) infections mediated by KPC type $\beta$-lactamases are creating a serious threat to our "last resort" antibiotics, the carbapenems. KPC $\beta$ lactamases are thus carbapenemases and are a subclass of Class A $\beta$-lactamases that have evolved to efficiently hydrolyze carbapenems and cephamycins which contain substitutions at the $\alpha$ position proximal to the carbonyl group that normally render these $\beta$-lactams resistant to hydrolysis. To investigate the molecular basis of this carbapenemase activity, we have determined the structure of KPC- 2 at $1.85 \AA$ resolution. The active site of KPC- 2 reveals the presence of a bicine buffer molecule which interacts via its carboxyl group with conserved active site residues S130, K234, T235, and T237; this likely resembles the interactions the $\beta$-lactam carboxyl moiety makes in the MichaelisMenten complex. Comparison of the KPC-2 structure with non-carbapenemases and previously determined NMC-A and SME-1 carbapenemase structures shows several active site alterations that are unique among carbapenemases. An outward shift of the catalytic S70 residue renders the active sites of the carbapenemases more shallow likely allowing easier access of the bulkier substrates. Further space for the $\alpha$-substituents is likely provided by shifts in N132 and N170 in addition to concerted movements in the postulated carboxyl binding pocket that might allow the substrates to bind in a slightly different angle to accommodate these $\alpha$-substituents. The structure of KPC-2 thus provides key insights into the carbapenemase activity of emerging Class A $\beta$-lactamases.
\end{abstract}

Carbapenems (imipenem, meropenem, and ertapenem) are the "last resort" $\beta$-lactam antibiotics for treating serious infections caused by multi-drug resistant gram-negative bacteria. Unfortunately, the acquisition of carbapenem-hydrolyzing $\beta$-lactamases by bacteria has resulted in a major threat to the clinical utility of these compounds $(1 ; 2)$. Currently the Klebsiella pneumoniae carbapenemases (KPC-type $\beta$-lactamases), are rapidly emerging as a major threat in the New York area (3-5), Pennsylvania (6), and internationally (7;8). Three KPC variants have been found so far (KPC-1,-2, and -3) of which KPC-1 and KPC-2 are almost indistinguishable, whereas KPC-3 exhibits different hydrolytic properties (9).

KPC carbapenemases belong to the Ambler class A $\beta$-lactamases (E.C. 3.5.2.6) $(10 ; 11)$. In common with other class A $\beta$-lactamase, these highly proficient class A enzymes have an efficient hydrolysis 'machinery' involving a catalytic serine residue (S70), which acylates $\beta$ -

Department of Biochemistry RT500, School of Medicine, Case Western Reserve University 10900 Euclid Avenue, Cleveland, OH 44106-4935, phone: (216) 368-8511, fax: (216) 368-3419, focco.vandenakker@case.edu.

Coordinates and structure factors for the KPC-2 structure have been deposited with the Protein Data Bank (PDB identifier 2OV5). 
lactam substrates, and a well-positioned deacylation water. S70 is involved in the nucleophilic attack at the carbonyl carbon of the substrate leading to cleavage of the bond with the $\beta$-lactam ring nitrogen for penicillin and other substrates (Figure 1). The deacylation water molecule is primed by E166 and N170 for nucleophilic attack of the $\beta$-lactam-acyl-enzyme intermediate and serves to liberate the $\beta$-lactam substrate from the active site by deacylation. Both acylation and deacylation involve a nucleophilic attack which is facilitated by a correctly positioned oxyanion hole (formed by the backbone nitrogens of residues 70 and 237) that attracts the carbonyl oxygen atom. These described active site elements are supported by a complex hydrogen bonding network involving a pair of conserved lysines (K37 and K234), N132, and S130.

Serine carbapenemases such as KPCs are unique among the class A $\beta$-lactamases because of their ability to hydrolyze $\beta$-lactams containing a substituent at the $\alpha$ position of the carbon atom in the $\beta$-lactam ring adjacent to the carbonyl $(12 ; 13)$. Examples of such readily hydrolyzed $\beta$ lactams by carbapenemases include imipenem (a carbapenem containing a $6 \alpha$-hydroxy-ethyl moiety) but also cefoxitin (a cephamycin containing a $7 \alpha-\mathrm{O}-\mathrm{CH}_{3}$ group)(Figure 1$)(9 ; 12 ; 13)$. Previous crystallographic studies of the class A serine carbapenemase NMC-A and SME-1 did not yield a unified conclusion regarding their carbapenemase phenotype. Interpretation of the NMC-A structure suggested a role for the slightly shifted position of N132 to perhaps accommodate these $\alpha$-substituents $(12 ; 14)$ whereas the SME-1 structure suggested a needed conformational change for catalysis and importance for the C69-C238 disulfide, being in close proximity of the active site (13). Mutagenesis studies of the C69-C238 residues in SME-1 confirmed the critical role of this disulfide bond, but its function was not limited to carbapenemase activity since its disruption caused loss of hydrolytic activity towards all $\beta$ lactam antibiotics (15). Based upon mutagenesis studies it was concluded that no single residue within the active site of SME-1 is solely responsible for carbapenemase activity and that this activity is likely due to multiple simultaneous substitutions and accompanying conformational rearrangements that prime the active site to accommodate and hydrolyze carbapenems and cephamycins (16).

The unresolved structural basis of this carbapenemase activity seen in class A enzymes and the urgent clinical threat presented by KPC $\beta$-lactamases found in Klebsiella, Enterobacter, and Salmonella spp. prompted our investigation of KPC-2. We present here the structure of KPC-2 determined to $1.85 \AA$ resolution. Our analysis of KPC-2 in comparison to other class A $\beta$-lactamase enzymes reveals alterations in active site topology with respect to noncarbapenemases such as SHV-1 and TEM-1 that could rationalize the structural basis of carbapenemase activity. Furthermore, the active site of KPC-2 contains a well-resolved bicine molecule with its carboxyl moiety occupying a space that is likely involved in recognizing the carboxyl moiety of $\beta$-lactams thus providing insights into substrate recognition.

\section{Materials and Methods}

The KPC-2 $\beta$-lactamase is expressed using the vector pBR322-catI-blaKPC-2 in E. coli DH10B cells (a kind gift of Dr. Fred Tenover, the Centers of Disease Control and Prevention, Atlanta GA). The plasmid containing bla $a_{\mathrm{KPC}-2}$ was described by Yigit et al (17) and has been confirmed by DNA sequencing. Transfected cells were grown in $500 \mathrm{ml}$ of Luria Bertaini (LB) broth containing chloramphenicol $20 \mathrm{ug} / \mathrm{ml}$ and grown for $18 \mathrm{hrs}$ and pelleted by centrifugation $(5000 \mathrm{~g} \times 15 \mathrm{~min})$. Cell pellets were resuspended in $20 \mathrm{mM}$ Tris- $\mathrm{Cl}, \mathrm{pH} 7.0$ and periplasmic proteins were released using stringent periplasmic fractionation with lysozyme and EDTA as previously described (18). The supernatant was filter sterilized, passed through phenylboronate column (MoBiTec), which has specific binding affinity for class A $\beta$-lactamases, and KPC-2 was eluted using $0.5 \mathrm{M}$ boronate/ $0.5 \mathrm{M} \mathrm{NaCl}, \mathrm{pH} 7.0$. The eluent was loaded on Superdex 75 size exclusion column and fractions containing nitrocefin-hydrolyzing activity were pooled 
and concentrated to $13 \mathrm{mg} / \mathrm{ml}$ in $10 \mathrm{mM}$ Tris- $\mathrm{HCl} \mathrm{pH} 7.6$ buffer (protein concentration was measured using Bradford method). The homogeneity of KPC-2 was estimated at $\sim 99 \%$ as assessed by SDS-PAGE. Carbapenemase activity was confirmed by hydrolysis of imipenem (imipenem $\lambda=299 ; \Delta \varepsilon=-9000 \mathrm{M}^{-1} \mathrm{~cm}^{-1}$ ).

Initial crystallization screens were carried out using the Grid PEG6000 Screen kit (Hampton Research Inc.) resulting in small needle crystal clusters. Crystallization conditions were optimized and diffraction quality KPC-2 crystals could be obtained using the sitting drop method and $0.7 \mu 1$ protein solution was mixed with $0.3 \mu \mathrm{l}$ reservoir solution and equilibrated against a reservoir solution containing 16\% PEG6000 in 0.1M bicine, pH 9.0. Crystals grew to full size in 3-14 days.

For crystallographic data collection, a single crystal was transferred to mother liquor containing $15 \%$ glycerol for 1 minute after which the crystal was flash frozen in liquid nitrogen. A $1.85 \AA$ resolution diffraction data set was collected at APS 19BM at $100 \mathrm{~K}$. The crystals belong to trigonal space group $\mathrm{P} 3_{1}$ with cell parameters $\mathrm{a}=116.245 \AA$, $\mathrm{b}=116.245 \AA$, $\mathrm{c}=52.001 \AA, \alpha=90^{\circ}$ $\mathrm{C}, \beta=90{ }^{\circ} \mathrm{C}, \mathrm{Q}=120^{\circ} \mathrm{C}$. The diffraction data were integrated and scaled using HKL2000 (19) (see Table 1 for processing statistics).

The KPC-2 structure was solved by molecular replacement using the program PHASER (20) with chain A of the SME-1 structure (PDB identifier 1DY6 (13)) as the initial search model. PHASER found 3 molecules in the asymmetric unit resulting in a solvent content of $47 \%$. The top solution underwent several rounds of REFMAC (21) refinement and manual model building with COOT (22) which resulted in an $\mathrm{R} / \mathrm{R}_{\text {free }}$ of $27.1 / 33.2 \%$. The inability to further improve the R-factors suggested a possible twinning problem which was confirmed using the Crystal Twinning Server (23). Subsequent refinement was carried out using CNS (24) with twinning operation " $h,-h-k,-l$ " and twinning fraction 0.420. Simulated annealing refinement followed by temperature factor refinement and manual model building lowered the R-factors considerably. No non-crystallographic symmetry restraints were used in refinement due to the relatively high resolution of the diffraction data. Electron density was of excellent quality for most of the residues (see Figure 2A for representative density near one of the active sites). Throughout the refinement, electron density for a bicine buffer molecule in each of the three KPC-2 molecules was apparent (Figure 2B). Therefore, three bicine buffer molecules were included in refinement as well as 294 water molecules and the final model containing residues 30-292 for each molecule (see Table 1 for additional details). The progress of crystallographic refinement was monitored using the program DDQ (25) and the final model quality was assessed using PROCHECK (26).

\section{Results}

The structures of three KPC-2 molecules present in the asymmetric unit are refined to an R/ $\mathrm{R}_{\text {free }}$ of $14.8 / 19.0 \%$ and $90 \%$ of the residues had phi-psi angles in the acceptable region of the Ramanchandran plot (Table 1 and Figure 3A). Each molecule contains one cis-peptide (residue 167 ) which is common amongst other class A $\beta$-lactamase structures. The three refined KPC-2 molecules in the asymmetric unit are very similar to each other as reflected by the low r.m.s.d. of 0.29-0.34 ̊ for superposition of all C $\alpha$ atoms (Figure 3B). The active site residues are also in very similar position as well. Since these three molecules are very similar, we will be limiting our discussion and analysis to KPC-2 subunit A.

The structure of KPC-2 contains two subdomains generating a cleft resulting in an overall fold that is expectedly similar to that of other class A $\beta$-lactamases (Figure 3A). One of the subdomains is largely $\alpha$-helical whereas the other subdomain contains a 5 -stranded $\beta$-sheet flanked by $\alpha$-helices. The cleft generated by these two subdomains harbors the active site 
containing the catalytic $\mathrm{S} 70$ residue and also harbors the deacylation water that is primed by interacting with E166 and N170 as well as S70 (Figure 4). The oxyanion hole formed by the backbone nitrogens of S70 and T237 is partially occluded by the side chain of S70 which is somewhat unusual for class A $\beta$-lactamases as will be discussed later. Adjacent to S70 is residue C69 which is involved in a disulfide bond with $\mathrm{C} 238$. This disulfide bond is characteristic for class A carbapenemases including NMC-A and SME (position 69 in class A enzymes usually is a $\mathrm{M}$ or other hydrophobic residue). Both conserved lysines (K73 and K234) are also present in the active site of KPC-2 as well as residue N132. The importance of these residues have been well established in class A $\beta$-lactamases (as reviewed in $(27 ; 28)$ ). The entrance of the active site harbors W105 and R220 situated on opposite sides of the active site (Figure 4).

The active site contains a serendipitous, though interesting finding in the form of a bound bicine buffer molecule. A bicine molecule is observed in the active site of all three KPC-2 molecules present in the asymmetric unit. The density is clearest for bicine in KPC-2 molecule A, with an average B-factor for bicine of $25 \AA^{2}$, and its omit electron density is depicted in Figure 2B. The $\mathrm{B}$-factors for bicine in molecules $\mathrm{B}$ and $\mathrm{C}$ refined to somewhat higher average temperature factors of 36 and $45 \AA^{2}$, respectively. Bicine (di(hydroxyethyl)glycine) is a zwitterionic compound and contains a tertiary amine, a carboxyl group and two hydroxyethyl groups (Figure 1). Its carboxyl moiety is involved in the majority of bicine's interactions in the active site by making hydrogen bonds with T235, T237, S130, a salt-bridge interaction with K234, a more distant $\sim 4 \AA$ electrostatic interaction with R220, and some water-mediated interactions (Figure 4). The two hydroxyethyl moieties of bicine do however not make direct hydrogen bonds with the protein (only a water-mediated interaction involving Wat3) but do provide hydrophobic interactions with W105. In addition, W105 could also be involved in a possible cation- $\pi$ interaction of bicine's tertiary amine group (Figure 4). Preliminary kinetic analysis showed that bicine had some detectable though weak inhibitory effect on KPC-2 activity (measured up to $900 \mathrm{mM}$ ) and we therefore estimate its affinity is likely around $0.1 \mathrm{M}$ range, close to the $100 \mathrm{mM}$ buffer concentration during the crystallization experiment.

\section{Discussion}

The structure of KPC-2 at 1.85 Å resolution provides a new understanding of the active site of a clinically important carbapenemase responsible for the widespread emergence of imipenemresistance in K. pneumoniae and other bacteria. Thus, the details revealed by our study are best appreciated in light of the structures of other serine class A $\beta$-lactamases. The precise mechanism that accounted for the carbapenemase phenotype in the class A $\beta$-lactamases, NMC-A and SME-1, was not precisely defined. The atomic structures of NMC-A and SME-1 when compared to TEM did not reveal a unifying structural basis for this enhanced catalytic activity $(12 ; 13)$. Nevertheless, the carbapenemase $v s$. non-carbapenemase hydrolytic differences are dramatic since the former can hydrolyze carbapenems with great efficiency: the $k_{\text {cat }} / K_{\mathrm{m}}$ for imipenem hydrolysis is only $2.4 \mathrm{mM}^{-1} \mathrm{~s}^{-1}$ for TEM-1 (29), non-detectable for SHV-1 (30), yet is 100-4,500 fold more efficient for carbapenemases with $k_{\text {cat }} / K_{\mathrm{m}}$ values of $300,440,1900$, and $11300 \mathrm{mM}^{-1} \mathrm{~s}^{-1}$ for KPC-2, SME-1, KPC-3, and NMC-A, respectively $(9 ; 12 ; 13)$. A similar trend is observed for the cephamycin cefoxitin which is also hydrolyzed more efficiently by the carbapenemases, compared to TEM-1 and SHV-1 $(9 ; 12 ; 13 ; 31)$. Compared to these Class A structures, the KPC-2 structure offers key insights into this novel kinetic behavior.

Superposition of the C $\alpha$ atoms of KPC-2 onto NMC-A, SME-1, SHV-1, and TEM-1 results in r.m.s.d. values of $0.81 \AA$, $0.80 \AA, 1.38 \AA, 1.20 \AA$, respectively (for $256,256,235$, and 240 superpositioned $\mathrm{C} \alpha$ atoms). Hence, KPC-2, SME-1, and NMC-A are quite similar in overall structure (Figure 5) with the largest deviations located distant from the active site (near loop residues 88 and 98, Figure 5A). The sequence conservation shows a similar trend as KPC-2 is 
52\% and 54\% sequence identical with SME- 1 and NMC-A, yet only shares 38\% and 36\% identity with SHV-1 and TEM-1, respectively. Most of the active site residues are remarkably conserved between class A carbapenemases and non-carbapenemases except for a few residues that are different in the latter (Figure 5B). These residues are T237 (is similar S in NMC-A and SME-1 yet is A in SHV-1 and TEM-1), H274 (M in SHV-1 and TEM-1), R220 (although an $\mathrm{R}$ is present in SHV-1 and TEM-1 it comes from residue R244 instead), and T216 (M in SHV-1 and TEM-1). T237, H274, and R220 are all near the carboxyl moiety of bicine suggesting an important role for these residues in that region of the active site, perhaps also interacting with the carboxyl moiety of the carbapenem and cephamycin substrates. Buried behind the active site is also the disulfide bond formed by $\mathrm{C} 69$ and C238 which is not present in SHV-1 and TEM-1. In addition to these residue differences, the active sites of the three carbapenemase structures reveal a number of conserved shifts with respect to the non-carbapenemases, SHV-1 and TEM- $1 \beta$-lactamases (Figure 5B, Table 2 and 3 ), suggesting a functional significance. These shifts are significant since they are all larger than the $0.2 \AA$ ESD coordinate errors reported for these structures (coordinate errors are listed in Table 2).

Comparing SHV-1 and TEM-1 vs. KPC-2, NMC-A, and SME-1, we observed that the following active site alterations are unique amongst the carbapenemases. Firstly, the decreased length of the pocket containing two water molecules Wat (deacylation water) and Wat2 (occupies temporarily oxyanion hole) stands out as distinctive amongst the carbapenemases. This length of this pocket is determined by the position of the OE2 atom of E166 and N atom of residue 237 which are located on opposite ends of this pocket each making one hydrogen bond with one of the water molecules. This space reduction in pocket length is quantified by the observed 0.5-1.9 ̊ shortening of the N:T237-OE2:E166 distance (Table 2). Residue E166 in itself is shifted by $0.3-0.9 \AA$ in the carbapenemases compared to non-carbapenemases (Table 3). This pocket is flanked by S70 whose relative repositioning of $0.5-0.8 \AA$ in carbapenemases (Table 3) further decreases the size of the pocket for the two waters as evidenced by the 0.5-1.4A reduction of the CA:S70-CA:237 distance in carbapenemases relative to the noncarbapenemases (Table 2). S70 is therefore positioned less deep in the active site in carbapenemases. Secondly, there is increased space adjacent to the water pocket by concurrent shifts of N132 and N170 in roughly opposite directions. This is measured by the $0.6-0.9 \AA$ increase in distance between the CA atoms of N170 and N132 for carbapenemases (Table 2). Thirdly, a reoriented carbonyl group of C238 due to C69-C238 disulfide bond and 1 residue insertion after $\mathrm{C} 238$ is observed. Lastly, the $0.4-0.8 \AA$ relative shift of the OG atom of S130 residue, the $0.6-1.7 \AA$ relative shift of the OG1 atom of Thr235, and the $0.8-1.5 \AA$ relative shift of residue T216 are also unique features amongst the carbapenemases (Table 3).

The change listed in our first observation result in decreased space for the usually present waters Wat 1 and Wat 2 and has a remarkable effect on the number of waters present in the active sites of the carbapenemases depending on the position of S70. In KPC-2, the side chain of S70 partially blocks the oxyanion hole and this structure therefore lacks Wat2 (Figure 5B). In SME-1, S70 points into the direction of the deacylation water cavity and this structure therefore lacks Wat1 yet does harbor the oxyanion hole Wat2. Only NMC-A has both Wat1 and Wat2 present in the active site since S70 has shifted somewhat in NMC-A less compared to the other two carbapenemases (Figure 5B). In non-carbapenemases such as SHV-1 and TEM-1, these waters are always present in their apo structures (Figure 5B). Since both cavities need to be available during catalysis (Wat2 cavity for the carbonyl oxygen atom of carbapenem during acylation and Wat 1 for subsequent deacylation), we speculate that S70 will likely adopt different conformations during catalysis to free these cavities in a successive manner. Increasing S70 movement is likely made possible by the more protruding S70 position in the active sites of carbapenemases (Figure 5B). 
The above listed changes have potential consequences for the ability of carbapenemases to hydrolyze imipenem and cefoxitin. First, we hypothesize that having S70 positioned in a more shallow position means that the $\beta$-lactam ring of carbapenems does not have to enter the catalytic cleft as deep which would normally be sterically hampered by the $6 \alpha$-substituent of carbapenems or $7 \alpha$-substituent of cephamycins. To illustrate this, we have used the structure of the cephalothin:AmpC Michaelis-Menten substrate complex complex and superimposed it onto KPC-2 (Figures 4 and 6)(32). Although AmpC is a Class C $\beta$-lactamase, it is by our knowledge the only known substrate:serine- $\beta$-lactamase complex and many key active site residues are conserved between Class $\mathrm{C}$ and Class A $\beta$-lactamases (i.e. S70, K73, N132, K234, G236, T235, and A/T237). This substrate complex superposition provides a good starting point for understanding the significance of the observed shifts with respect to carbapenems and cephamycin hydrolysis, in particular since cephalothin is structurally related to the cephamycin cefoxitin as it only lacks the $7 \alpha-\mathrm{O}-\mathrm{CH}_{3}$ moiety (Figure 1).

The superposition revealed that the carboxyl moiety of cephalothin is in a similar position as the carboxyl moiety of bicine, an observation that also strengthens the relevance of bicine's active site interactions. The observed shift of S70 being positioned less deep in the active site would allow the cefoxitin to approach the active site at a slightly different angle such that its $7 \alpha-\mathrm{O}-\mathrm{CH}_{3}$ moiety shifts to a wider area of the active site to minimize steric clashes (Figure $6 \mathrm{~A})$. In addition to this approximate in-plane reorientation of cefoxitin, cefoxitin is postulated to undergo an additional movement perpendicular to the first rotation (Figure 6B). This second rotation is likely due to the concerted shifts of S130 and T235 (Figure 5B) which are postulated to provide key anchoring hydrogen bonds with the carboxyl group of cefoxitin as extrapolated from the initial superposition of the AmpC complex with KPC-2 (Figure 4), the bicine interactions with KPC-2, and that the carboxyl moiety of cefoxitin forms a hydrogen bond with T316 in AmpC which is the structural equivalent of T235 in KPC-2 (32). In addition, the carboxyl moiety of the inhibitor $6 \alpha$-(hydroxypropyl)-penicillanate when bound to NMC-A forms similar interactions with T235, S237, S130, and K73 although this a covalently acylenzyme complex and not a Michaelis-Menten complex (14). Both S130 and T235 are shifted such that the carboxyl group and the rest of the ligand might undergo a counter-clock wise rotation as indicated in Figure 6B. The active site is wide enough to accommodate such a reorientation of cefoxitin which likely results in providing additional space for the $7 \alpha-\mathrm{OCH}_{3}$ group. An interesting observation is that the carboxyl moiety of bicine, and presumably also cefoxitin, also interacts with T237, in addition to S130 and T235 (Figures 4 and 6B). The importance for the $\mathrm{O} \gamma$ atom at position 237 has been noted since only a $\mathrm{S}$ or $\mathrm{T}$ at this position could maintain carbapenemase activity (16;33). Loss of this hydroxyl in SME-1 leads to a 5fold decrease in $k_{\text {cat }}$ for imipenem hydrolysis (33). This suggests that the $\mathrm{O} \gamma$ of residue 237 could indeed be critical for orienting cefoxitin, and imipenem, via interaction with their carboxyl moieties thereby improving the catalytic efficiency of their hydrolysis.

Residues in the immediate vicinity of T237 are R220 and H274 with which it forms a $2.7 \AA$ hydrogen bond and $3.4 \AA$ van der Waals interaction, respectively. As noted above, all three residues are changed in SHV-1 and TEM-1 further emphasizing the importance of this region for carbapenemase activity. R220 residue provides, in addition to its hydrogen bond with T237, also a $\sim 4 \AA$ electrostatic interaction and a water-mediated interaction with the carboxyl moiety of bicine. In KPC-3, residue $\mathrm{H} 274$ is mutated to a tyrosine which leads to a $\sim 6$-fold enhancement in $k_{\text {cat }} / K_{\mathrm{m}}$ for imipenem compared to KPC-2 (and KPC-1) (9). Both R220 and $\mathrm{H} 274$ are therefore postulated to have an effect on substrate binding either electrostatically, or indirectly via correctly positioning either T237 and/or the Wat 3 water molecule (see Figure 4). In summary, the ability of carbapenemases to accommodate $\alpha$-substituents present in carbapenems and cephamycins is postulated to result from multiple active site adjustments such that these substrates can access the active site in a somewhat different orientation thereby repositioning their $\alpha$-substituents to a wider part of the active site. Shifts of nearby N170 and 
N132 in particular likely provide additional room as well. The active site shifts are supported by conserved residue changes in carbapenemases in particular near the postulated carboxyl binding site to provide additional direct or indirect interaction strength.

The perimeter of the active site of carbapenemases contains an additional residue that merit consideration. This residue is W105 which in the SME- 1 carbapenemase and other class A $\beta$ lactamases has been found to have a strong preference for aromatic residues (16). This aromatic residue at position 105 could provide favorable stacking interactions with the carbapenem substrate in the Michaelis-Menten complex $(34 ; 35)$ as is also evident from the cefoxitin superposition (Figures 4 and 6). SME-1 and NMC-A contains a histidine at this position whereas KPC-2 contains a tryptophan but even non-carbapenemases usually have an aromatic residue at this position (Figure 5B). It is a possibility that either a $\mathrm{H}$ or $\mathrm{W}$ at this position could provide an hydrogen bond with the substrate in addition to the postulated stacking interaction. Residues T217 and P104 (F in NMC-A and Y in SME-1) are located a bit more distant compared to W105 yet could potentially play a role in interacting with the different substituents of carbapenems and cephamycins.

In addition to the postulated changes in carbapenemases to reorient the carbapenem and cephamycin substrates such that their $\alpha$-substituents can be accommodated in a productive Michaelis-Menten complex, carbapenemases have also evolved to not get inhibited by longlived reaction intermediates of these substrates. For example, TEM-1 has been shown to react with imipenem albeit with low affinity. However, imipenem's acyl-enzyme intermediate becomes trapped in the active site of TEM-1 due to displacement of the deacylation water and the repositioning of the $\beta$-lactam carbonyl outside the oxyanion hole, interacting with S130 instead (36). This non-productive deacylation configuration is likely due to imipenem's $6 \alpha-$ hydroxy-ethyl moiety which makes a hydrogen bond with N132. In the carbapenemases, such a trapped covalently-bound imipenem configuration is likely avoided by the simultaneous shift of N132 and S70 in somewhat opposite directions, limiting such N132-mediated intermediatestabilizing interactions and favoring efficient carbapenem hydrolysis (Figure 5B). Thus, carbapenemases are not only able to accommodate the bulkier carbapenems in the initial binding step but also avoid being inhibited by undesired long-lived intermediates. This could be aided by the postulated increased flexibility of S70. The S70 flexibility could even have a role in allowing the carbapenems and cephamycins to readjust to accommodate the $\alpha$ substituent group during the initial Michaelis-Menten complex.

In summary, we present the structure of KPC-2, a clinically important carbapenemase present in $K$. pneumoniae and other nosocomial pathogens. This analysis provides insight into the unique properties of this $\beta$-lactamase and explains its ability to hydrolyze imipenem and other carbapenems, and cephamycin, antibiotics that constitute our "last line" of defense against $\beta$ lactam resistant bacteria. Our findings also demonstrate that apparently subtle changes in active site topology ( 0.5 to $0.8 \AA$ A relocation of the catalytic S70) and other shifts in conserved amino acid positions in class A $\beta$-lactamases have a profound effect on substrate specificity likely by allowing the substrates to bind in a slightly different angle to alleviate steric hindrance. The process of new $\beta$-lactam drug discovery must anticipate these unexpected consequences of protein remodeling. It is important to recall that the carboxyl moiety of bicine in KPC-2 is in an almost identical position as the carboxyl moiety of our previously designed SA2-13 inhibitor when bound to SHV-1 (37). This suggests that the inhibition strategy of trans-enamine stabilization using carboxyl-linker penam sulfone derivatives could potentially be applicable to carbapenemases. 


\section{Acknowledgments}

We thank Dr. Fred Tenover (CDC) for the pBR322-catI-blaKPC-2 construct. We thank the personnel at the SBC beamline 19-BM at Advanced Photon Source for help with data collection and use of their facilities.

RAB is supported by the Merit Review Program and NIH grant R01AI063517-01. JMT is supported by NIH Grant T32 GM07250 and the Case Medical Scientist Training Program. FvdA is supported by NIH grant AI062968.

\section{Abbreviations used}

KPC, Klebsiella pneumoniae carbapenemase; r.m.s.d., root-mean-square-deviation.

\section{References}

1. Babic M, Hujer AM, Bonomo RA. What's new in antibiotic resistance? Focus on beta-lactamases. Drug Resist. Updat 2006;9:142-156. [PubMed: 16899402]

2. Paterson DL. Resistance in gram-negative bacteria: Enterobacteriaceae. Am. J. Infect. Control 2006;34:S20-S28. [PubMed: 16813978]

3. Bratu S, Mooty M, Nichani S, Landman D, Gullans C, Pettinato B, Karumudi U, Tolaney P, Quale J. Emergence of KPC-possessing Klebsiella pneumoniae in Brooklyn, New York: epidemiology and recommendations for detection. Antimicrob. Agents Chemother 2005;49:3018-3020. [PubMed: 15980389]

4. Woodford N, Tierno PM Jr. Young K, Tysall L, Palepou MF, Ward E, Painter RE, Suber DF, Shungu D, Silver LL, Inglima K, Kornblum J, Livermore DM. Outbreak of Klebsiella pneumoniae producing a new carbapenem-hydrolyzing class A beta-lactamase, KPC-3, in a New York Medical Center. Antimicrob. Agents Chemother 2004;48:4793-4799. [PubMed: 15561858]

5. Bratu S, Landman D, Haag R, Recco R, Eramo A, Alam M, Quale J. Rapid spread of carbapenemresistant Klebsiella pneumoniae in New York City: a new threat to our antibiotic armamentarium. Arch. Intern. Med 2005;165:1430-1435. [PubMed: 15983294]

6. Pope J, Adams J, Doi Y, Szabo D, Paterson DL. KPC type beta-lactamase, rural Pennsylvania. Emerg. Infect. Dis 2006;12:1613-1614. [PubMed: 17176590]

7. Naas T, Nordmann P, Vedel G, Poyart C. Plasmid-mediated carbapenem-hydrolyzing beta-lactamase $\mathrm{KPC}$ in a Klebsiella pneumoniae isolate from France. Antimicrob. Agents Chemother 2005;49:44234424. [PubMed: 16189140]

8. Navon-Venezia S, Chmelnitsky I, Leavitt A, Schwaber MJ, Schwartz D, Carmeli Y. Plasmid-mediated imipenem-hydrolyzing enzyme KPC-2 among multiple carbapenem-resistant Escherichia coli clones in Israel. Antimicrob. Agents Chemother 2006;50:3098-3101. [PubMed: 16940107]

9. Alba J, Ishii Y, Thomson K, Moland ES, Yamaguchi K. Kinetics study of KPC-3, a plasmid-encoded class A carbapenem-hydrolyzing beta-lactamase. Antimicrob. Agents Chemother 2005;49:47604762. [PubMed: 16251324]

10. Ambler RP, Coulson AF, Frere JM, Ghuysen JM, Joris B, Forsman M, Levesque RC, Tiraby G, Waley SG. A standard numbering scheme for the class A beta-lactamases. Biochem. J 1991;276(Pt 1):269270. [PubMed: 2039479]

11. Yigit H, Queenan AM, Anderson GJ, Domenech-Sanchez A, Biddle JW, Steward CD, Alberti S, Bush K, Tenover FC. Novel carbapenem-hydrolyzing beta-lactamase, KPC-1, from a carbapenemresistant strain of Klebsiella pneumoniae. Antimicrob. Agents Chemother 2001;45:1151-1161. [PubMed: 11257029]

12. Swaren P, Maveyraud L, Raquet X, Cabantous S, Duez C, Pedelacq JD, Mariotte-Boyer S, Mourey L, Labia R, Nicolas-Chanoine MH, Nordmann P, Frere JM, Samama JP. X-ray analysis of the NMCA beta-lactamase at 1.64-A resolution, a class A carbapenemase with broad substrate specificity. J. Biol. Chem 1998;273:26714-26721. [PubMed: 9756914]

13. Sougakoff W, L'Hermite G, Pernot L, Naas T, Guillet V, Nordmann P, Jarlier V, Delettre J. Structure of the imipenem-hydrolyzing class A beta-lactamase SME-1 from Serratia marcescens. Acta Crystallogr. D. Biol. Crystallogr 2002;58:267-274. [PubMed: 11807251] 
14. Mourey L, Miyashita K, Swaren P, Bulychev A, Samama JP, Mobashery S. Inhibition of the NMCA beta-lactamase by a penicillanic acid derivative and the structural bases for the increase in substrate profile of this antibiotic resistance enzyme. J. Am. Chem. Soc 1998;120:9382-9383.

15. Majiduddin FK, Palzkill T. Amino acid sequence requirements at residues 69 and 238 for the SME-1 beta-lactamase to confer resistance to beta-lactam antibiotics. Antimicrob. Agents Chemother 2003;47:1062-1067. [PubMed: 12604542]

16. Majiduddin FK, Palzkill T. Amino acid residues that contribute to substrate specificity of class A beta-lactamase SME-1. Antimicrob. Agents Chemother 2005;49:3421-3427. [PubMed: 16048956]

17. Yigit H, Queenan AM, Rasheed JK, Biddle JW, Domenech-Sanchez A, Alberti S, Bush K, Tenover FC. Carbapenem-resistant strain of Klebsiella oxytoca harboring carbapenem-hydrolyzing betalactamase KPC-2. Antimicrob. Agents Chemother 2003;47:3881-3889. [PubMed: 14638498]

18. Hujer AM, Hujer KM, Bonomo RA. Mutagenesis of amino acid residues in the SHV-1 beta-lactamase: the premier role of Gly238Ser in penicillin and cephalosporin resistance. Biochim. Biophys. Acta 2001;1547:37-50. [PubMed: 11343789]

19. Otwinowski Z, Minor W. Processing of X-ray diffraction data collected in oscillation mode. Methods Enzymol 1997;276:307-326.

20. McCoy AJ, Grosse-Kunstleve RW, Storoni LC, Read RJ. Likelihood-enhanced fast translation functions. Acta Crystallogr. D. Biol. Crystallogr 2005;61:458-464. [PubMed: 15805601]

21. Murshudov GN, Vagin AA, Dodson EJ. Refinement of macromolecular structures by the maximumlikelihood method. Acta Crystallogr. D. Biol. Crystallogr 1997;53:240-255. [PubMed: 15299926]

22. Emsley P, Cowtan K. Coot: model-building tools for molecular graphics. Acta Crystallogr. D. Biol. Crystallogr 2004;60:2126-2132. [PubMed: 15572765]

23. Yeates TO. Detecting and overcoming crystal twinning. Methods Enzymol 1997;276:344-358. [PubMed: 9048378]

24. Brunger AT, Adams PD, Clore GM, DeLano WL, Gros P, Grosse-Kunstleve RW, Jiang JS, Kuszewski J, Nilges M, Pannu NS, Read RJ, Rice LM, Simonson T, Warren GL. Crystallography \& NMR system: A new software suite for macromolecular structure determination. Acta Crystallogr. D 1998;54:905-921. [PubMed: 9757107]

25. van den Akker F, Hol WG. Difference density quality (DDQ): a method to assess the global and local correctness of macromolecular crystal structures. Acta Crystallogr. D Biol. Crystallogr 1999;55(Pt 1):206-218. [PubMed: 10089411]

26. Laskowski RA, MacArthur MW, Moss DS, Thornton JM. PROCHECK - a program to check the stereochemical quality of protein structures. J. Appl. Cryst 2001;26:283-291.

27. Matagne A, Lamotte-Brasseur J, Frere JM. Catalytic properties of class A beta-lactamases: efficiency and diversity. Biochem. J 1998;330(Pt 2):581-598. [PubMed: 9480862]

28. Minasov G, Wang X, Shoichet BK. An ultrahigh resolution structure of TEM-1 beta-lactamase suggests a role for Glu166 as the general base in acylation. J. Am. Chem. Soc 2002;124:5333-5340. [PubMed: 11996574]

29. Raquet X, Lamotte-Brasseur J, Bouillenne F, Frere JM. A disulfide bridge near the active site of carbapenem-hydrolyzing class A beta-lactamases might explain their unusual substrate profile. Proteins 1997;27:47-58. [PubMed: 9037711]

30. Poirel L, Heritier C, Podglajen I, Sougakoff W, Gutmann L, Nordmann P. Emergence in Klebsiella pneumoniae of a chromosome-encoded SHV beta-lactamase that compromises the efficacy of imipenem. Antimicrob. Agents Chemother 2003;47:755-758. [PubMed: 12543688]

31. Neu HC. Beta-lactamase stability of cefoxitin in comparison with other beta-lactam compounds. Diagn. Microbiol. Infect. Dis 1983;1:313-316. [PubMed: 6321094]

32. Beadle BM, Trehan I, Focia PJ, Shoichet BK. Structural milestones in the reaction pathway of an amide hydrolase: substrate, acyl, and product complexes of cephalothin with AmpC beta-lactamase. Structure. (Camb. ) 2002;10:413-424. [PubMed: 12005439]

33. Sougakoff W, Naas T, Nordmann P, Collatz E, Jarlier V. Role of ser-237 in the substrate specificity of the carbapenem-hydrolyzing class A beta-lactamase Sme-1. Biochim. Biophys. Acta 1999;1433:153-158. [PubMed: 10446368] 
34. Doucet N, De Wals PY, Pelletier JN. Site-saturation mutagenesis of Tyr-105 reveals its importance in substrate stabilization and discrimination in TEM-1 beta-lactamase. J. Biol. Chem 2004;279:46295-46303. [PubMed: 15326193]

35. Bethel CR, Hujer AM, Hujer KM, Thomson JM, Ruszczycky MW, Anderson VE, Pusztai-Carey M, Taracila M, Helfand MS, Bonomo RA. Role of Asp104 in the SHV beta-lactamase. Antimicrob. Agents Chemother 2006;50:4124-4131. [PubMed: 16982784]

36. Maveyraud L, Mourey L, Kotra LP, Pedelacq JD, Guillet V, Mobashery S, Samama JP. Structural basis for clinical longevity of carbapenem antibiotics in the face of challenge by the common class A beta-lactamases from the antibiotic-resistant bacteria. J. Am. Chem. Soc 1998;120:9748-9752.

37. Padayatti PS, Sheri A, Totir MA, Helfand MS, Carey MP, Anderson VE, Carey PR, Bethel CR, Bonomo RA, Buynak JD, van den AF. Rational design of a beta-lactamase inhibitor achieved via stabilization of the trans-enamine intermediate: 1.28 A crystal structure of wt SHV-1 complex with a penam sulfone. J. Am. Chem. Soc 2006;128:13235-13242. [PubMed: 17017804] 


\section{Carbapenem}

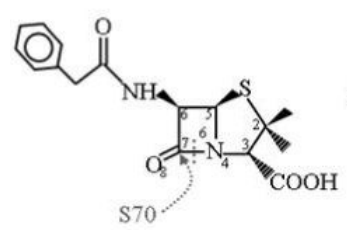

Penicillin G

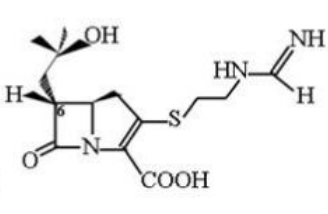

Imipenem
Cephamycin

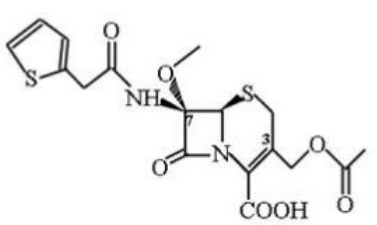

Cefoxitin

\section{Cephalosporin}

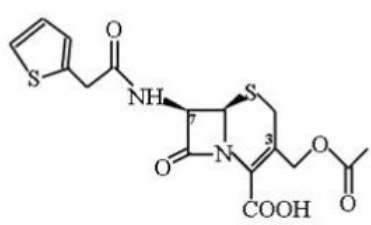

Cephalothin<smiles>O=C(O)CN(CCO)CCO</smiles>

Bicine

Figure 1.

Schematic diagram of penicillin G, a carbapenem (imipenem), cephamycin (cefoxitin), a cephalosporin (cephalothin), and bicine. The bond that is broken during the acylation step involving S70's nucleophilic attack (curved grey dotted arrow) is shown only for penicillin G (straight dotted grey line between $\mathrm{C} 7$ and N4). 

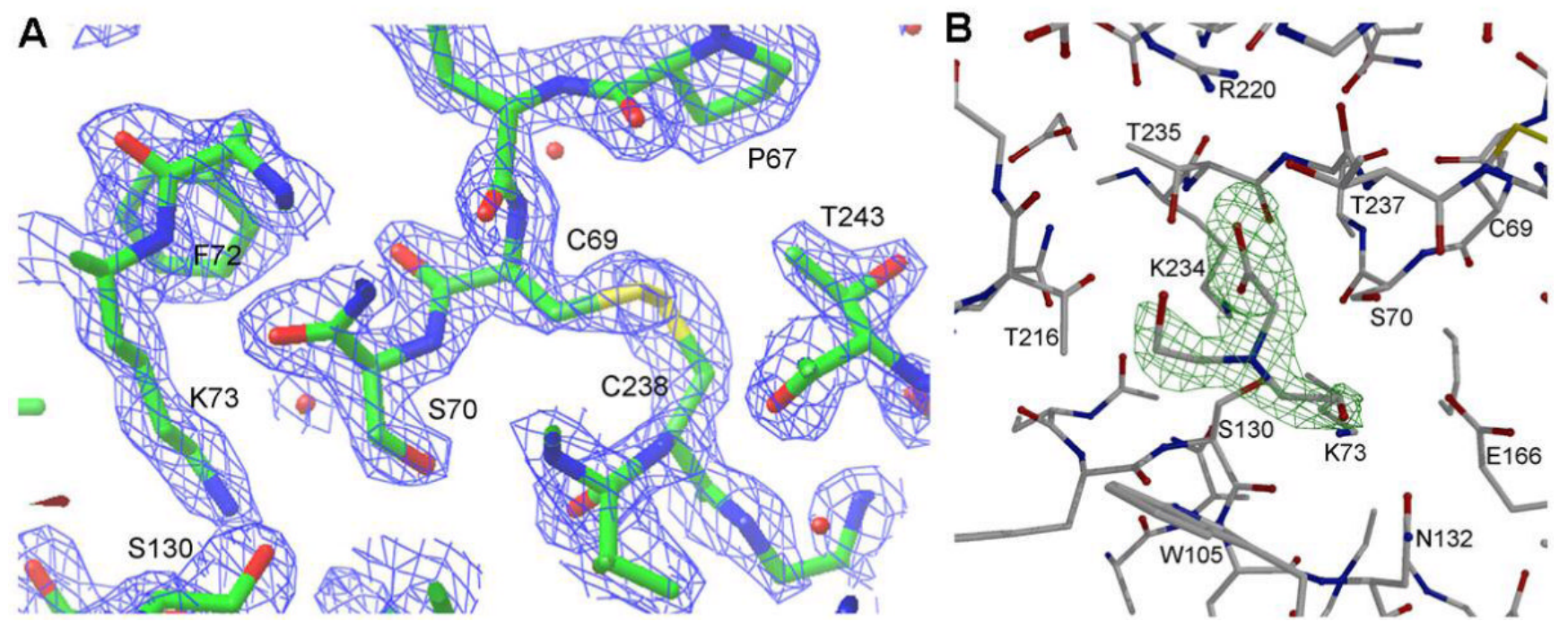

Figure 2.

(A) Electron density of a region in the vicinity of the active site of KPC-2. $1.85 \AA$ resolution | $\mathrm{Fo}|-| \mathrm{Fc} \mid$ simulated annealing omit map contoured at $2.5 \sigma$ is depicted. (B) Electron density for bicine in the active site of KPC-2. $|\mathrm{Fo}|-|\mathrm{Fc}|$ simulated annealing omit electron density is contoured at $2.5 \sigma$. 

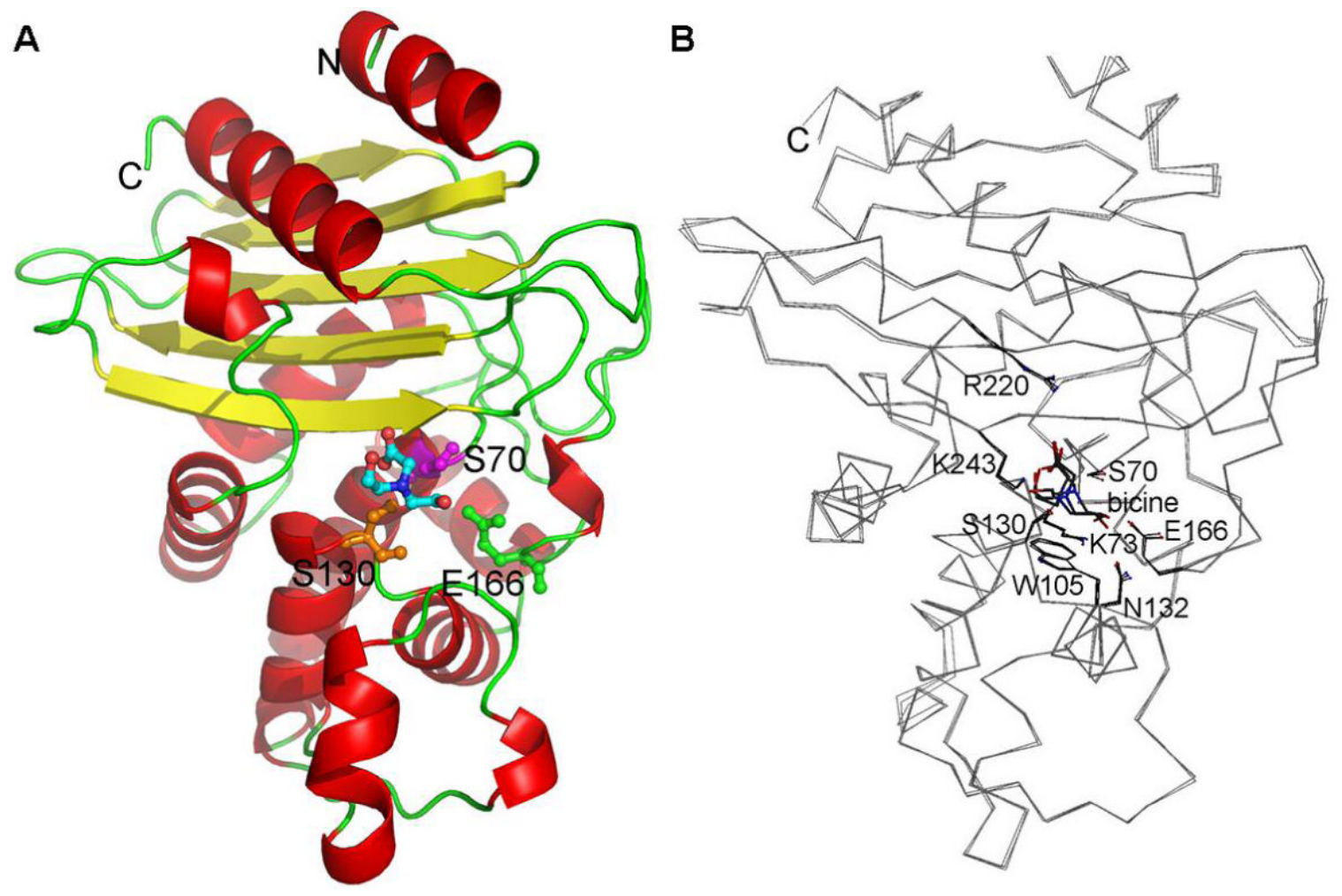

Figure 3.

(A) Structure of KPC-2 $\beta$-lactamase. The secondary structure elements are depicted as yellow ( $\beta$-strands), red ( $\alpha$-helices), and coil (green). To indicate the position of the active site, the positions of the catalytic residues S70 (magenta), S130 (orange), and E166 (green) are labeled. Bicine is color coded by atom type with the carbon atoms shown in blue. (B) Superposition of three KPC-2 molecules present in the asymmetric unit. The three KPC-2 molecules are depicted in a $\mathrm{C} \alpha$ trace. Active site residues and bicine molecules are shown in stick representation and labeled accordingly. 


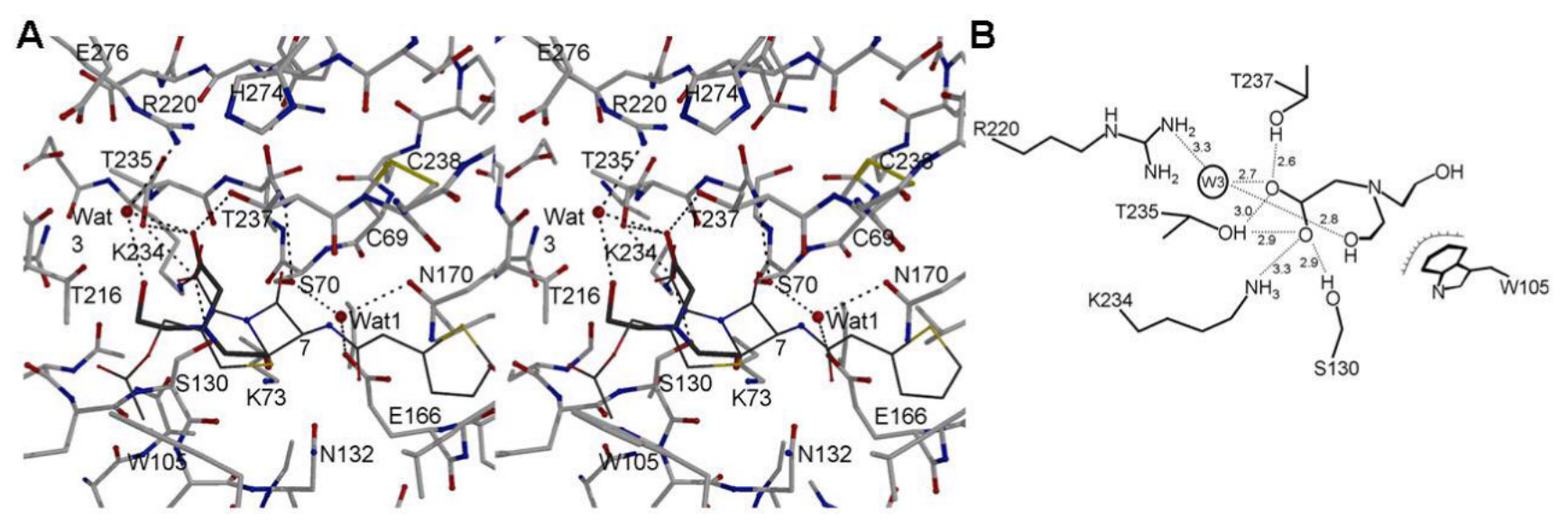

Figure 4.

(A) Stereo figure depicting the active site of KPC-2 with bicine and a superpositioned cephalosporin. Bicine (thick dark sticks) and the cephalosporin cephalothin (thin dark sticks) are depicted. The position of cephalothin was obtained by superimposing the AmpC:cephalothin substrate complex (PDBid 1KVL (32)) with KPC-2. The C $\alpha$ atoms used in the superpositioning are KPC-2 residues 69-73, 132, and 235-238 onto AmpC residues 63-67, 152, and 316-319 (superposition resulted in an r.m.s.d. of $0.67 \AA$ for the $10 \mathrm{C} \alpha$ atoms). Hydrogen bonds with bicine are shown as dashed lines. The carbon position of cephalothin in which cefoxitin has a $7 \alpha-\mathrm{OCH}_{3}$ substituent is labeled with a ' 7 '. (B) Schematic diagram of interactions of bicine in the active site of KPC-2. Hydrogen bonds are depicted by dashed lines; van der Waals interactions with W105 are shown by a curved line with perpendicular lines. Hydrogen bond distances are listed in $\AA$. 
A

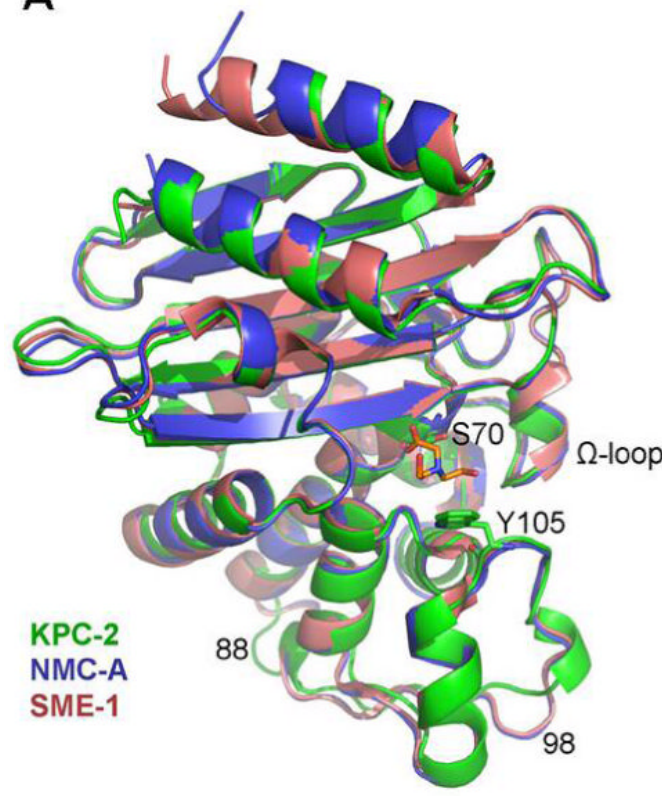

B

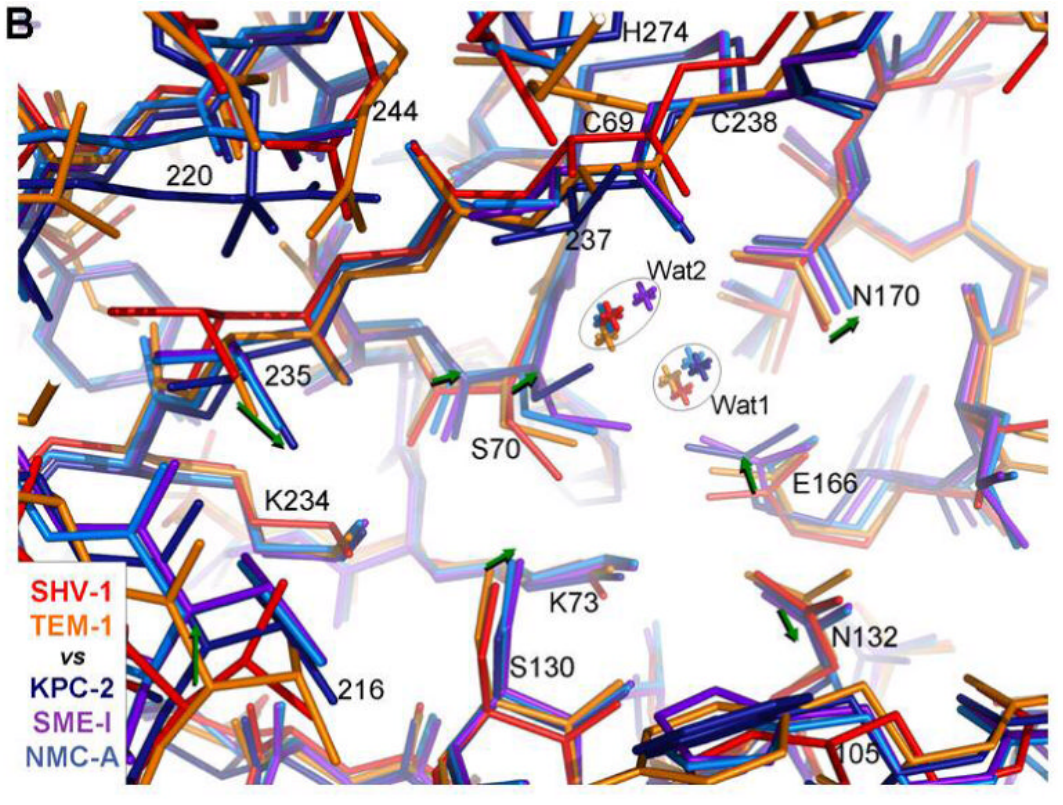

Figure 5.

(A) Superposition of class A carbapenemases. The position of the active site is indicated by depicting residues S70 and Y105 as well as the flanking $\Omega$-loop and bicine (in stick representation with cold colored carbon atoms). A couple of loops are deviating signicantly between KPC-2 and NMC-A and SME-1 and are labeled (near residues 88 and 98). (B)

Superposition of the active sites of the carbapenemases and non-carbapenemase $\beta$-lactamases. Superpositioned are KPC-2 (dark blue), NMC-A (light blue), and SME-1 (magenta) and noncarbapenemases SHV-1 (red) and TEM-1 (orange). Green arrows highlight common shifts comparing the carbapenemase and non-carbapenemase structures. Two key clusters of waters are circled: the deacylation water primed by E166 and N170 (Wat1) and the water occupying the oxyanion hole formed by backbone nitrogens of residues 70 and 237 (Wat2). The following $11 \mathrm{C} \alpha$ atoms were used to superposition the active sites of the $\beta$-lactamases: $69,70,73,105$, 130, 132, 166, 170, 234, 236, and 237. 


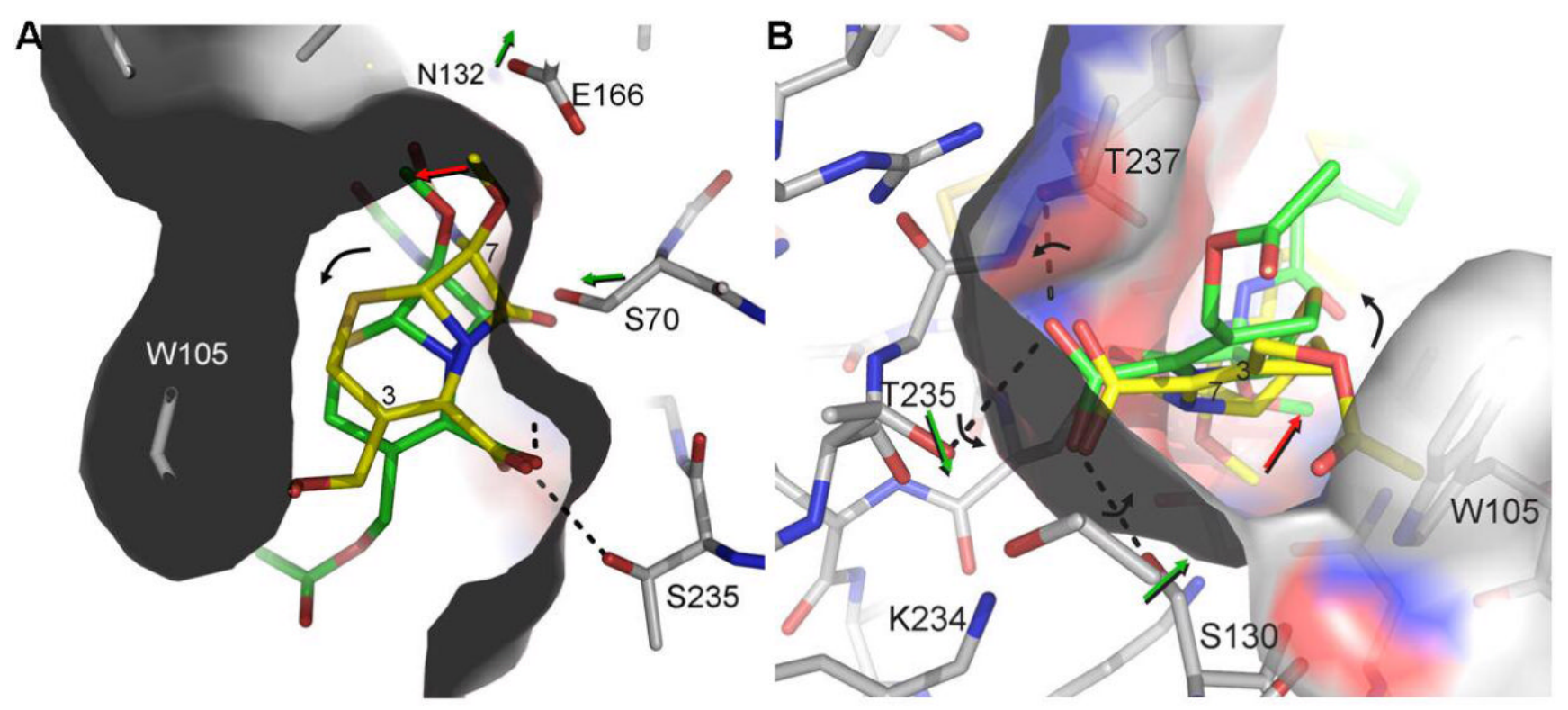

Figure 6.

Active site adjustments of KPC-2 and their postulated role in accommodating cefoxitin. The initial position of cefoxitin is obtained after superpositioning of the KPC-2 and

AmpC:cephalothin active sites and adding the $\mathrm{O}-\mathrm{CH}_{3}$ moiety to cephalothin's $7 \alpha$ position to form cefoxitin (yellow). Cefoxitin was reoriented (green), guided by observed active site readjustments, to alleviate steric clashes with its $7 \alpha-\mathrm{O}-\mathrm{CH}_{3}$ moiety. In addition, the conformation of its 3 -substituent was adjusted as well to alleviate steric clashes. A, view from the bottom of the active site. Observed key shifts in KPC-2 relative to non-carbapenemases are indicated by green arrows. The shift of residue N132 (faint in background) likely provides additional space for the $7 \alpha-\mathrm{O}-\mathrm{CH}_{3}$ substituent of cefoxitin. The observed shift of $\mathrm{S} 70$ to a more outward position allows a less deep active site penetration of the $\beta$-lactam ring of cefoxitin. This could result in a ligand reorientation (curved arrow) such that the $7 \alpha-\mathrm{O}-\mathrm{CH}_{3}$ moiety shifts (red arrow) thereby further alleviating steric clashes. B, side view obtained by an approximate $90^{\circ}$ rotation along the horizontal axis compared to the view in A. This view shows the postulated effects of the concerted shifts of T235 and S130 (green arrows) in possibly reorienting the carboxyl moiety (in direction of the small curved arrows), and the rest of cefoxitin (in the direction of the larger curved arrow), in a counter-clock wise fashion to reposition its $7 \alpha-\mathrm{O}-\mathrm{CH}_{3}$ group (red arrow) to a wider region of the active site to prevent steric clashes. The presence of the Ser/Thr at position 237 in carbapenemases could provide an additional stabilizing hydrogen bond for the carboxyl moiety of cefoxitin to aid in this postulated reorientation (SHV-1 and TEM-1 have an Ala at this position). The described shifts and reorientation for cefoxitin are also postulated to occur for carbapenems. 
Table 1

Data collection and refinement statistics for KPC-2 structure

Data Collection

Space group

Unit cell dimensions $(\AA)$

Wavelength $(\AA)$

Resolution $(\AA)$

Redundancy

Data cut-off $(\sigma)$

Unique reflections

$\langle\mathrm{I}\rangle \mid\langle\sigma(\mathrm{I})\rangle$

$\mathrm{R}_{\text {merge }}(\%)$

Completeness (\%)

Refinement

Resolution range ( $\AA$ )

Atoms in asymmetric unit

$\mathrm{R}$-factor (\%)

$\mathrm{R}$-free (\%)

RMSD deviations from ideality

Bond lengths $(\AA)$

Angles $\left({ }^{\circ}\right)$

Average temperature factors $\left(\AA^{2}\right)$

Protein

Bicine

Waters

Ramanchandran plot statistics

Residues in -most favored regions -additional

allowed regions

disallowed regions
$\mathrm{P} 3_{1}$

116.25116 .2552 .009090120

0.97

$30-1.85(1.92-1.85)$

3.2

-3.0 (default)

64,107

$19.7(2.3)$

$6.4(34.4)$

$95.6(90.8)$

$30-1.85(1.93-1.85)$

6,174

$14.9(24.9)$

$19.0(26.0)$

0.0065

1.29

25.6

35.3
26.6

$\begin{array}{llll}90.2 \% & 9.2 \% & 0.6 \% & 0 \%\end{array}$ 


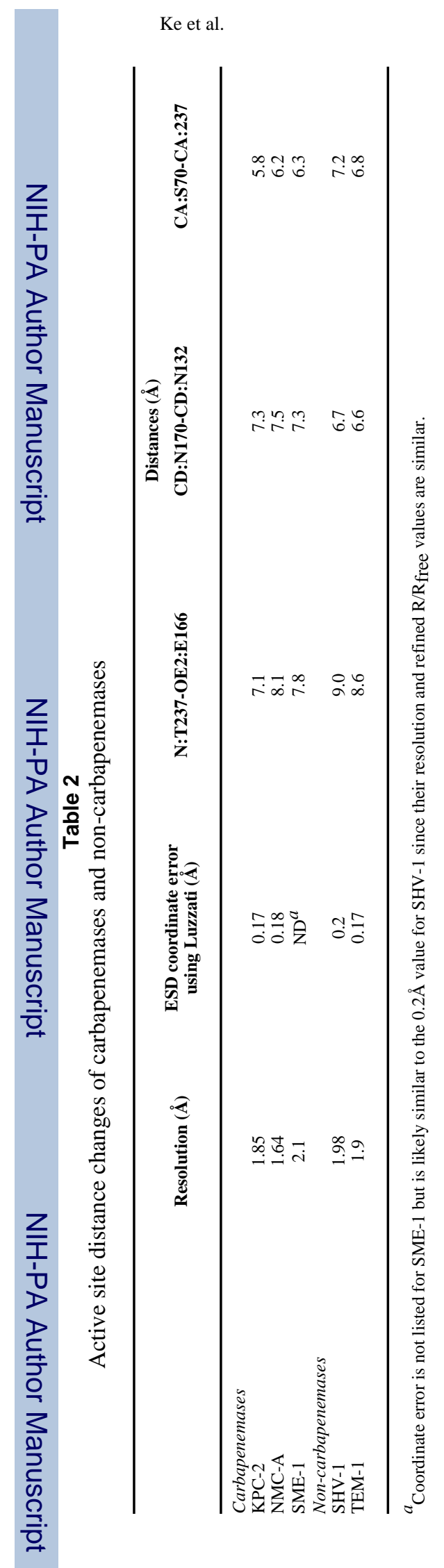

Page 18 


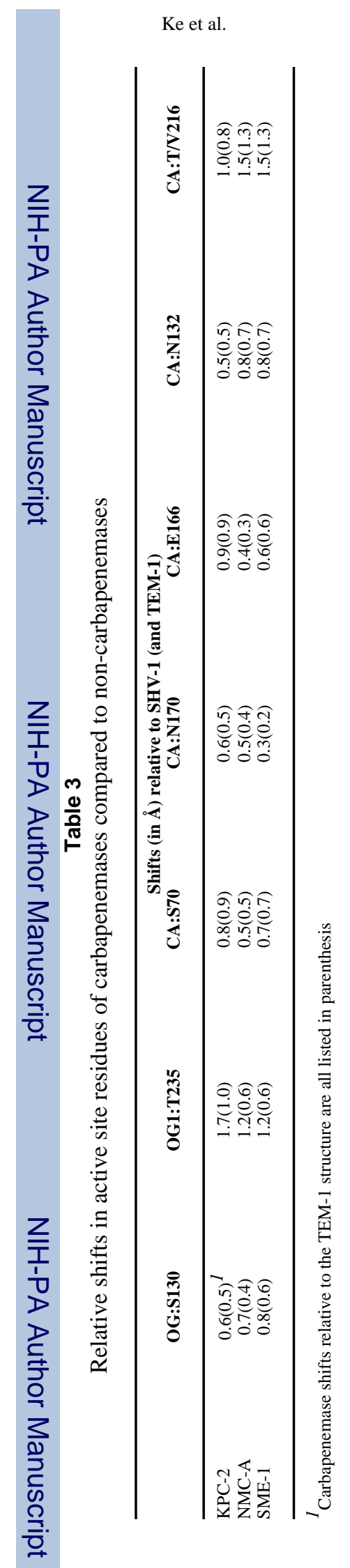

Page 19

Biochemistry. Author manuscript; available in PMC 2008 December 4. 